heart failure. The EST gene was cloned and expressed in E. coli; the recombinant protein is a non-disulfide linked homotrimer with a monomer molecular weight of $33 \mathrm{kDa}$ in both solution and crystalline state, indicating that these ESTs function as trimers. EST hydrolyzed DL-MATI to produce DAT with the degree of conversion of $49.5 \%$ and an enantiometric excess value $97.2 \%$ at optimum $\mathrm{pH}$ $8 \sim 10$ and temperature $57 \sim 67{ }^{\circ} \mathrm{C}$. The crystal structure of EST has been determined by X-ray diffraction to a resolution $1.6 \AA$, confirming that EST is a member of the $\alpha / \beta$ hydrolase fold superfamily of enzymes and includes a catalytic triad Ser97, Asp227 and His256. The active site is located approximately in the middle of the molecule at the end of a pocket $\sim 12 \AA$ deep. The EST-DL-MATI complex structure has also been determined and shows that the oxyanion hole can form by peptide $\mathrm{NH}$ groups of Thr98 and Trp31 that form hydrogen bonds with the carbonyl oxygen of the DL-MATI. The EST can hydrolyze methyl ester group without affecting the acetylthiol ester moiety in DL-MATI. The examination of substrate specificity of EST toward other linear esters revealed that the enzyme showed specific activity toward methyl esters and that it recognized the configuration at the $\mathrm{C}-2$ position. The knowledge of substrate specificity, molecular recognition and structure of a substrate-binding site of EST is useful for enzymatic engineering for broader ester substrates.

[1] Elmi, F., Lee, H.-T., Huang, J.-Y., Hsieh, Y.-C., Wang, Y.-L. Chen, Y.-J., Shaw, S.-Y., and Chen, C.-J. J. Bactiol., 2005, 187, 8470.

[2] Grirrane, A.; Pastor, A.; Galindo, A.; Ienco, A.; Mealli, C. Chemm. Commun. 2003, 512 .

\section{MS05 P04}

How structural features of MsrBs shed lights to the catalytic mechanism. Fanomezana M. Ranaivoson ${ }^{\mathrm{a}}$, Fabrice Neiers $^{\mathrm{b}}$, Sandrine Boschi-Muller ${ }^{\mathrm{b}}$, Guy Branlant ${ }^{\mathrm{b}}$, André Aubry ${ }^{\mathrm{a}}$ and Frédérique Favier ${ }^{\mathrm{a}}$

${ }^{\mathrm{a}} L C M 3 B, \quad{ }^{\mathrm{b}}$ MAEM, Nancy-Université, Nancy, France. E-mail: moutse.ranaivoson@lcm3b.uhp-nancy.fr

\section{Keywords: catalytic mechanisms, substrate binding,} methionine sulfoxide reductase

The thioether side-chain of methionine residues oxidizes easily to sulfoxide in vivo, especially under oxidative stress conditions. To maintain the cellular viability, organisms possess methionine sulfoxide (MetSO) reductases (Msrs), enzymes devoted to the repair of such oxidized proteins. To efficiently reduce the two possible stereoisomers of the sulfoxide function, two classes of Msrs have evolved: MsrA, specific to Met-S-SO, and MsrB specific to Met- $R$-SO [1]. The characterized catalytic mechanism for both MsrA and MsrB is based on the reactivity of two or three cysteine (Cys) residues and can be divided according to two major steps: a reductase step in which the substrate is reduced, and a recycling step needed by the enzyme to recover its initial active state [2]. The first step ends with the oxidation of the enzyme to a sulfenic acid intermediate. In the second step, the reacting Cys form an internal disulfide bond which necessitates the intervention of a reducing partner such as thioredoxin to terminate the recycling process. In the case of MsrBs, the known structure from Neisseria gonorrhoeae [3] shows a spatial disposition of the active site apparently optimized for the execution of both the first and the second steps. But the analysis of this structure combined with sequence alignments reveal that in other MsrBs where the reacting Cys are distributed differently, structural differences may appear in particular to allow the disulfide bond formation during the second step.

Here will be presented a series of crystallographic structures of MsrBs from two different organisms, retracing some steps of the catalytic mechanism. From the observation of these structures, some of the structural origins of the efficiency of the catalysis will be discussed, with regard to the deductions made with the structure from $N$. gonorrhoeae. In addition, the comparison between the two organisms of the current study will highlight the differences in the conformational behavior of the two MsrBs during the mechanism, due to the relative positioning of their Cys.

[1] Grimaud, R., Ezraty, B., Mitchell, J. K., Lafitte, D., Briand, C., Derrick, P. J., Barras, F., J Biol Chem, 2001., 276(52), 48915.

[2] Boschi-Muller, S., Olry, A., Antoine, M., Branlant, G., Biochim Biophys Acta, 2005. 1703(2), 231.

[3] Lowther, W. T., Weissbach, H., Etienne, F., Brot, N.

Matthews, B. W., Nat Struct Biol, 2002. 9(5), 348.

\section{MS05 P05}

Yellow lupine pathogenesis-related protein as a reservoir for cytokinins Humberto Fernandes*1, Anna Bujacz $^{2}$, Oliwia Pasternak ${ }^{1}$, Grzegorz Bujacz ${ }^{1,2}$, Michal Sikorski ${ }^{1}$, Mariusz Jaskólski ${ }^{1,3}{ }^{1}$ Center for Biocrystallographic Research, Inst. of Bioorg. Chem., Pol. Acad. Sci., Poznan, Poland. ${ }^{2}$ Technical Univ. of Lodz, Poland. ${ }^{3}$ Faculty of Chemistry, A. Mickiewicz Univ., Poznan,Poland. E-mail: humberto@man.poznan.pl

Keywords: plant pathogenesis-related proteins, plant hormones, cytokinins

Plants, forced to grow in harsh conditions and exposed to pathogenic activity, have developed several means of defense. Besides establishing a physical barrier by strengthening their cell wall, plants also produce antibiotic compounds called phytoalexins and accelerate cell death to suppress the spread of infection. Furthermore, the expression of a number of proteins is induced by various types of pathogens, or by chemicals such as ethylene or salicylic acid. These proteins, designated PR (pathogenesis-related), are grouped into seventeen classes according to their biological activity and sequence homology. PR proteins of class 10 , which are coded by multigene families, are small $(17 \mathrm{kDa})$, slightly acidic, and cytosolic. The main feature of their three-dimensional structure is a seven-stranded antiparallel $\beta$-sheet surrounding a long $\mathrm{C}$-terminal $\alpha$-helix, with a large cavity created between these two structural elements. Although PR-10 proteins are abundant in plants, their physiological role still remains unknown. One hypothesis implicates PR10 proteins in cytokinin binding. Cytokinins act as versatile hormones in regulation of plant development and growth. Two groups of cytokinins are known, adenine derivatives, such as zeatin, which are of natural origin, and synthetic urea derivatives, such as CPPU or DPU. Our crystallographic data obtained for a zeatin complex of a yellow lupine PR-10 protein have demonstrated the protein's unusual ability to bind as many as three zeatin ligands in the internal cavity. The high data resolution and excellent quality of the electron density maps allowed modeling of the zeatin molecules with atomic accuracy. Recently, the same PR-10 protein has been crystallized in complex with the urea-type cytokinins, DPU and CPPU. 